\title{
The design of a breadboard Cryogenic Optical Delay Line for DARWIN
}

\author{
T. van den Dool*a ${ }^{* a}$ F. Kamphues ${ }^{\mathrm{a}}$, B. Fouss ${ }^{\mathrm{b}}$, K. Henrioulle ${ }^{\mathrm{b}}$, \\ P.P. Kooijman ${ }^{\mathrm{c}}$, M.Visser ${ }^{\mathrm{d}}$, G. Velsink ${ }^{\mathrm{d}}$, K. Fleury $^{\mathrm{e}}$ \\ ${ }^{a}$ TNO TPD, Stieltjesweg 1, 2628 CK, Delft, THE NETHERLANDS \\ ${ }^{\mathrm{b}}$ Micromega-Dynamics, Rue des Chasseurs Ardennais, B-4031, Angleur (Liège), BELGIUM \\ 'SRON, Sorbonnelaan 2, 3584 CA, Utrecht, THE NETHERLANDS \\ ${ }^{\mathrm{d}}$ Dutch Space, Newtonweg 1, 2333 CP, Leiden, THE NETHERLANDS \\ ${ }^{\mathrm{e}} \mathrm{CSL}$, Parc Scientifique du Sart Tilman, Angleur (Liège), BELGIUM
}

\begin{abstract}
TNO TPD, in cooperation with Micromega-Dynamics, SRON, Dutch Space and CSL, has designed a compact breadboard cryogenic delay line for use in future space interferometry missions. The work is performed under ESA contract in preparation for the DARWIN mission. The breadboard (BB) delay line is representative of a future flight mechanism, with all materials and processes used being flight representative. The delay line has a single stage voice coil actuator for Optical Path Difference (OPD) control, driving a two-mirror cat's eye. Magnetic bearings provide frictionless and wear free operation with zero-hysteresis. Overall power consumption is below the ESA specification of $2.5 \mathrm{~W}$. The power dissipated on the optical bench at $40 \mathrm{~K}$ is considerably less than the maximum allowable $25 \mathrm{~mW}$.

The BB delay line will be build in the second half of 2004. The manufacturing and assembly phase is followed by a comprehensive test program, including functional testing at $40 \mathrm{~K}$ in 2005 . The tests will be carried out by Alcatel Space and SAGEIS-CSO.
\end{abstract}

Keywords: optical delay line, ODL, cryogenic, DARWIN, active magnetic bearings, nanopositioning, aperture synthesis

\section{INTRODUCTION}

TNO TPD and its partners have a long history in developing delay lines, with a large range of strokes and dimensions. Stroke lenghts varied from a few micron with subnanometer OPD stability (e.g. for the ASTRIUM Nulling Breadboard) to 60 meter and $7 \mathrm{~nm}$ RMS OPD stability (ESO VLTI delay line developed by Dutch Space and TNO TPD).

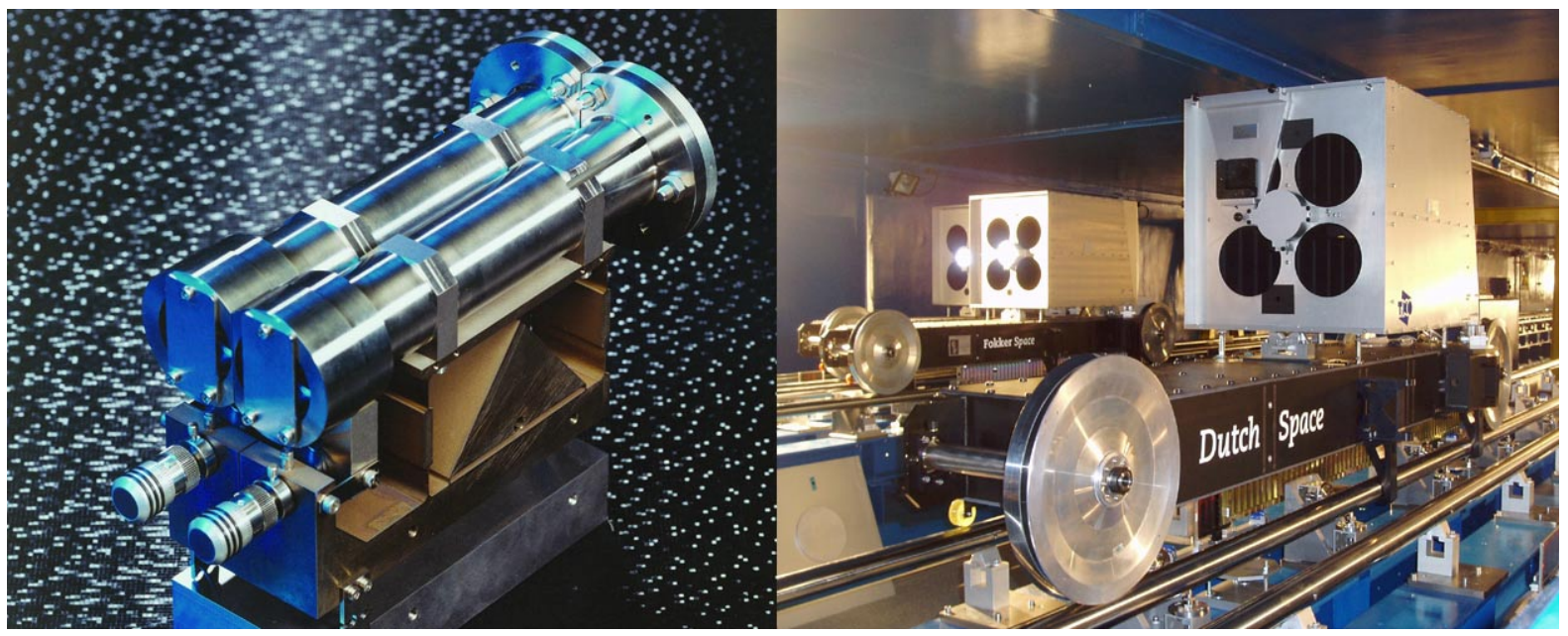

Fig. 1. Delay Lines for Nulling Breadboard

Fig. 2. VLTI Delay Lines

*dendool@tpd.tno.nl; phone 31-15-26 92 457; fax 31-15-26 92 111; www.tpd.tno.nl 
The main requirements for the breadboard DARWIN Optical Delay Line (ODL) are given in table 1 [1].

\begin{tabular}{|c|c|}
\hline Operational temperature/pressure & $40 \mathrm{~K}$ \\
\hline Pressure & $10^{-6} \mathrm{mbar}$ \\
\hline Launch loads & 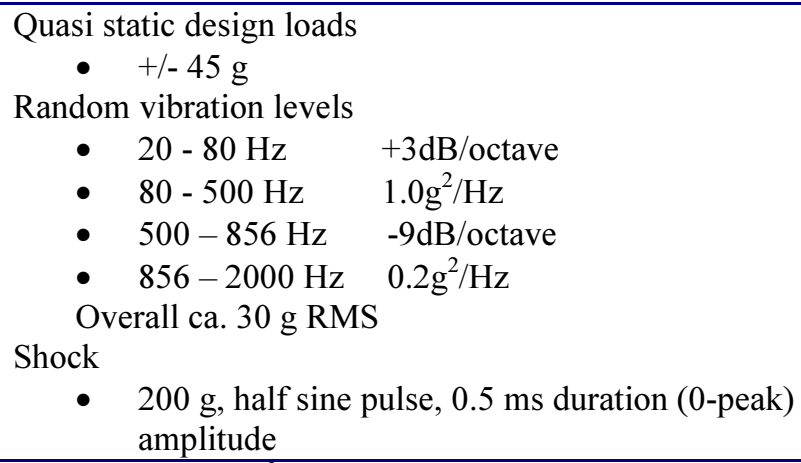 \\
\hline Microvibrations environment & $\begin{array}{l}\text { Amplitude } 7.5 \mathrm{~nm}^{2} / \mathrm{Hz} \\
\text { Cut-off frequency }(-3 \mathrm{~dB} \text { point }) 10 \mathrm{~Hz} \\
\text { Slope }-20 \mathrm{~dB} / \mathrm{dec}\end{array}$ \\
\hline ODL stability & $<1 \mathrm{~nm}$ RMS \\
\hline ODL stroke & $20 \mathrm{~mm}$ \\
\hline Dimensions & $<300 \times 100 \times 100 \mathrm{~mm}$ \\
\hline Mass & $<10 \mathrm{~kg}$ (target: $<6 \mathrm{~kg}$ ) \\
\hline Overall power dissipation & $<2.5 \mathrm{~W}$ \\
\hline Power dissipation in ODL & $<25 \mathrm{~mW}$ \\
\hline Optical beam diameter & $>25 \mathrm{~mm}$ \\
\hline Output beam tilt (over the full actuation range) & $<0.24 \mu \mathrm{rad}$ \\
\hline Wavelength range & $0.45-20 \mu \mathrm{m}$ \\
\hline Wavefront distortion & $<\lambda / 20 \operatorname{RMS}(\lambda=633 \mathrm{~nm})$ \\
\hline Coating manufacturing reproducibility & $\begin{array}{l}\text { Relative spectral response } \\
\qquad \quad<10^{-4} \\
\text { Chromatic phase differences } \\
\text { • OPD contribution }<0.1 \mathrm{~nm} \text { RMS } \\
\text { Relative polarization } \\
\text { - } \quad \text { Rotation }<0.1^{\circ} \\
\text { - } \quad \text { Ellipticity }<0.1^{\circ}\end{array}$ \\
\hline Transmission & $>94 \%$ \\
\hline Design lifetime & 10 years \\
\hline
\end{tabular}

Table 1. Optical Delay Line main requirements

In addition to the key requirements listed above, other critical items in DARWIN, that affect the design of the optical delay line are:

- The output rate of Fringe Sensor with respect to the DARWIN optical bench disturbance spectrum. If the disturbance spectrum exceeds half of the output rate of the Fringe Sensor, an additional sensor for OPD control will be required in DARWIN (e.g. a laser metrology system)

- Straylight

- Contamination of the optical surfaces

- Ground testing requirements (e.g. with a higher disturbance spectrum, or with the spacecraft in a specific orientation) 
A typical ODL system breakdown is given in figure 3.

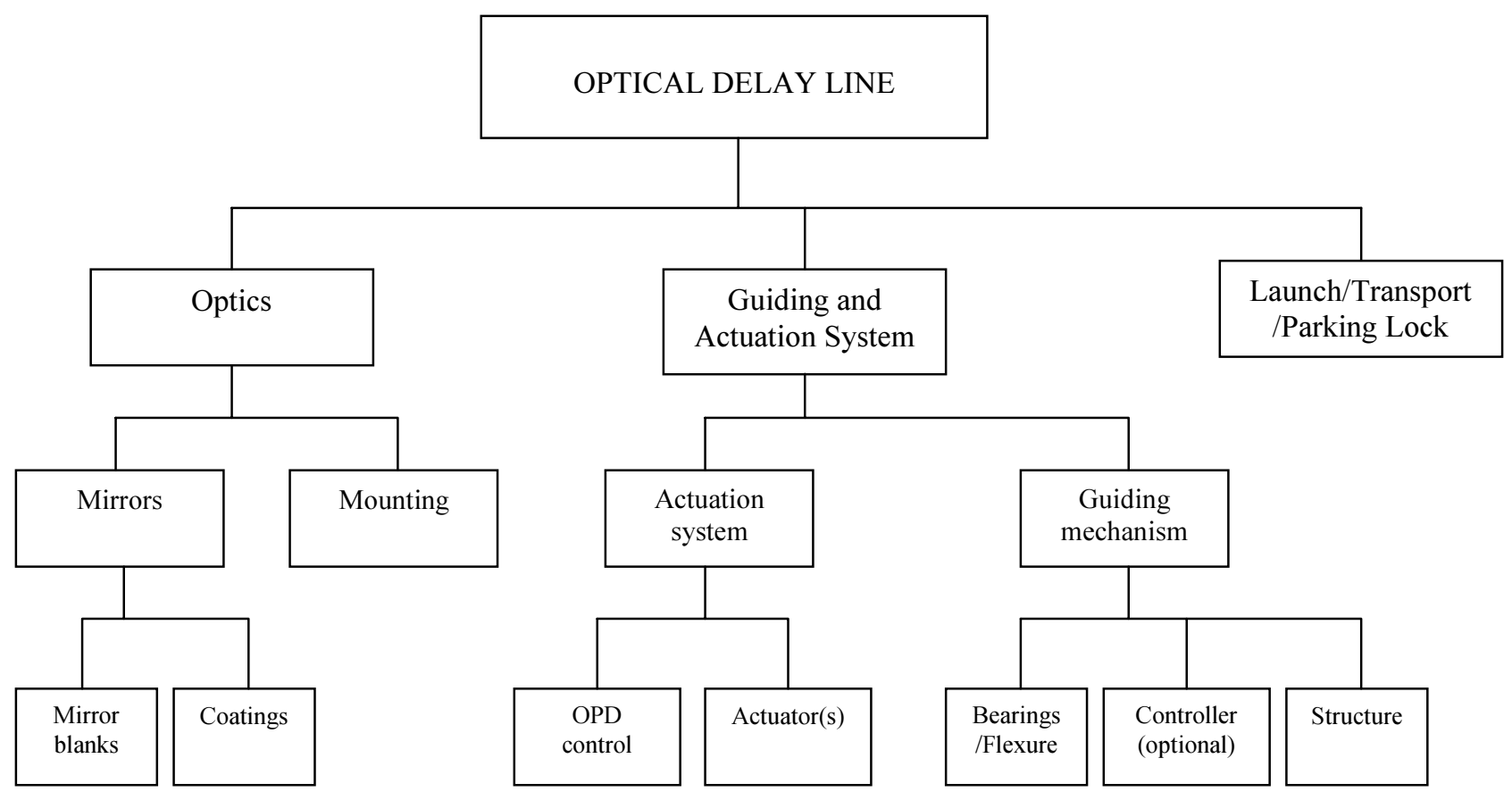

Fig. 3. Optical Delay Line system breakdown

Within this study, the responsibilities for the design and development are divided as follows:

- TNO TPD - Project management, systems engineering, optical design and OPD control

- Micromega-Dynamics - Guiding system development

- $\quad$ SRON - Actuator development and cryogenic consultancy

- CSL - Coating engineering and 40K TV facility

- Dutch Space - System modelling and 120K TV facility

- Alcatel - DARWIN mission level consultancy

The design and manufacturing phase will be followed by a comprehensive verification program, including functional testing at $40 \mathrm{~K}$. These tests will be carried out by Alcatel Space and SAGEIS-CSO.

\section{CONCEPT SELECTION}

An extensive trade off study has been performed to select the best concept for the DARWIN ODL.

For each building block of the ODL (see figure 3) a number of design options were reviewed, and its performance carefully assessed or analysed.

Note: the detailed design of a Launch Lock is not part of this study, but an assessment has been done at conceptual level. 


\subsection{Optical configuration trade-off}

Four concepts were investigated:

- Roof Top, i.e. two planar mirrors placed perpendicular with respect to each other.

- Corner Cube, i.e. three planar mirrors placed perpendicular with respect to each other.

- Two-mirror Cat's Eye, i.e. a combination of a focusing mirror and a planar mirror that is placed at the focus of that system.

- Three-mirror Cat's Eye, i.e. a combination of a focusing system (consisting of two mirrors) and a planar mirror that is placed at the focus of that system.

A roof top configuration was rejected because of its sensitivity to tilt in one direction.

A corner cube is not preferred because of its relative high angle of incidence and the possible introduction of polarisation errors. It also lacks the capability for pupil imaging, something that may be required for DARWIN in the future.

A cat's eye is the preferred configuration for DARWIN, because it has none of the disadvantages above and results in a very compact design. A two-mirror cat's eye was preferred over a three mirror cat's eye, because although it is slightly longer, it is the only configuration to fit in the $100 \times 100 \mathrm{~mm}$ cross section.

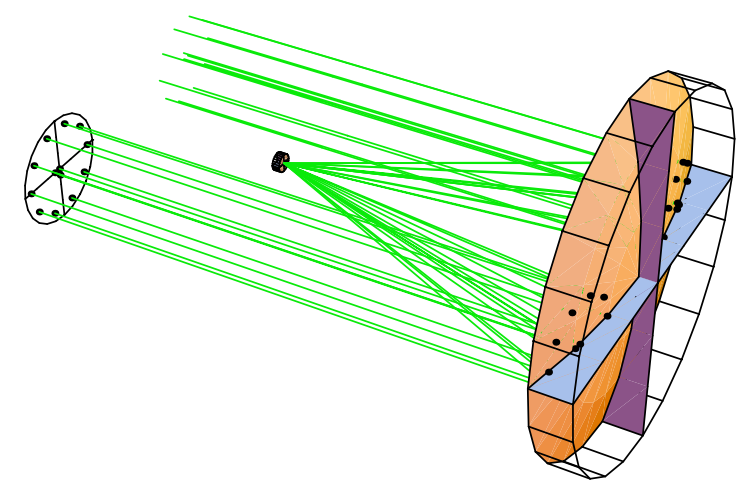

Fig. 4. Two-mirror Cat's Eye model

A number of reflective coatings were analysed. Gold and protected Silver were the two most suitable candidates.

With the stringent requirements on coating manufacturing reproducibility and lifetime, a single layer of Gold was preferred over a more complex and sensitive multi-layer protected Silver coating. The slightly lower transmission of Gold in the Fringe Sensor band is acceptable.

\subsection{Guiding system trade-off}

A number of options for the guiding mechanism are available:

- air/hydraulic bearing

- ball or roller bearing

- flexure

- magnetic bearing 
Air bearings and hydraulic bearing are considered unsuitable for space missions. Although air bearings are being used in some state of the art lithography systems (in vacuum), the possibility of leakage and contamination make them unsuitable for a mission like DARWIN.

A ball bearing system shows considerable stick/slip. It also shows considerable hysteresis when the direction of movement is reversed (as is the case in a DARWIN configuration, where the delay line works around a central position). This behaviour makes it difficult to control the OPD variations with just one actuator. Also the wear and lubrication are a concern for a long duration space mission, especially when they are constantly used over a very short stroke.

A flexure has none of the disadvantages above. However, for a $10 \mathrm{~mm}$ stroke, the dimensions may exceed the required envelope of $100 \times 100 \times 300 \mathrm{~mm}$. A flexure is also very complex (mechanically) and sensitive to temperature variations. A spring stiffness compensation is required to keep the power dissipation on the optical bench below $25 \mathrm{~mW}$.

Although relatively new, magnetic bearings fulfil all the guiding requirements. Micromega-Dynamics has demonstrated with the MABE platform, that zero friction and hysteresis, extremely low power dissipation, non-wear and high reliability can be successfully achieved with adaptive magnetic bearings.

The system is straightforward, with a sensor, permanent magnet and a set of active coils. Control can be either with simple local analogue electronics, or more complex central digital control.

In the last case, the control can be implemented in the same processor as used for OPD control.

Conclusion: a guiding system based on magnetic bearings with centralised digital control is the preferred solution for DARWIN.

\subsection{Actuation and OPD control trade-off}

The ODL shall be able to operate in three modes:

- Fringe acquisition (mm range): to scan over the whole ODL range in order to find the coherence length range where fringes appear (interferences).

- Zero path difference locking ( $\mu \mathrm{m}$ range): to scan within the fringe range (coherence length of the light source) in order to find the zero path difference, where a constructive interference occurs (destructive in case an achromatic $\pi$-phase shifter has been introduced in one of the arms of the interferometer) for all wavelengths.

- $\quad$ Path stabilization and tracking (nm range): to compensate the OPD variations due to optical path disturbances during the whole observation time (several hours), maintaining highly stable the required deep nulling value.

There are two levels of dynamic response requirements, corresponding to the above functions:

- In path stabilization and tracking mode, the limited output rate of the fringe tracker will not allow a high OPD control bandwidth, but low noise electronics will be required.

- However, on the other side of the operational modes, the dynamics for Fringe acquisition are more demanding. A step profile with short settling time and zero overshoot, and thus high peak accelerations, may be required. These requirements are driving for the dimensioning of the actuator and the implementation of the OPD control.

\subsubsection{Actuation trade-off}

TNO TPD applies a minimum-number-of-stages philosophy to all delay lines it develops. A smaller number of actuation stages simplifies the OPD control algorithm and improves the optical quality of the outgoing beam.

For a relatively short stroke, such as for DARWIN (20 mm optical), OPD control can be achieved with a single actuator. With a single stage delay line, the relative movement between mirrors can be prevented. This results in lower tilt and lateral displacement of the outgoing beam, which is another major benefit.

In order to enable single stage OPD actuation, the delay line needs to have:

- low moving mass

- low friction variations (e.g. stick/slip, bearing noise, power and signal wire torques)

- low hysteresis

- linear behaviour 
A single stage configuration is preferred over a dual stage configuration, providing the requirements for the guiding system can be met.

In a single stage delay line with high OPD stability $(<1 \mathrm{~nm})$ it is essential not to have wires to the moving part. An actuator with the electrical connections on the static part is preferred.

A number of actuators that comply with this requirement have been considered:

- Inchworm

- Voice Coil

- Linear Motor

The maturity of cryogenic inchworms is currently very low. They are also non contact-free and issue of glitches cannot be properly assessed at this stage. Wear and expected lifetime are another concern and therefore the inchworm is rejected as a candidate

There is very little difference between the performance of a voice coil and a linear motor. For the given mechanical stroke of $10 \mathrm{~mm}$, a voice coil has a small margin over a linear motor, with respect to the force to mass ratio. The amplifier electronics are also less complex, and a voice coil is the preferred solution for this application.

\subsubsection{OPD control trade-off}

The control can be implemented in several ways:

- Analogue

- Digital

- $\quad$ Fixed

- Self-calibrating/re-programmable

- Adaptive

Here an adaptive controller means that a (partial) controller redesign takes place on a sample-to-sample basis by using on-line measurements. A self-calibrating controller is fixed for most of the time but it's coefficients can be recalculated using off-line measurements and control design algorithms. This enables near optimal control performance even in case of changes in transfer functions, e.g. due to changes in temperature.

An assessment of the performance of the different type of controllers shows:

- For high performance, the controller is often a complex transfer function, which is difficult to implement accurately in analogue electronics. This especially holds for higher frequencies where the plant (actuator to sensor transfer function) is complex.

- For a high performance, the disturbance spectrum should be accurately known. The exact disturbance spectrum in DARWIN will not be known until the constellation has been designed, tested, launched and deployed. It would be advantageous in this case to have a controller that measures the disturbance spectrum and tunes the controller accordingly.

- The disturbances may also change over time, for instance due to a change in conditions (e.g. temperature changes, on-board failures of micro-thrusters, relays, etc.), and only an adaptive, or re-programmable controller would be able to cope with this.

- An (adaptive or re-programmable) digital controller requires relatively large processing power (and corresponding electrical power dissipation).

- The stability (amplitude and phase margins) of an adaptive controller is difficult to prove in a qualification program.

Typical space qualified Digital Signal Processor (DSP) boards dissipate in the order of $15 \mathrm{~W}$. However, using a radiation tolerant Field Programmable Gate Arrays (FPGA), or dedicated Application Specific Integrated Circuit (ASIC), the power dissipation could be reduced to well below the specified value $(<2.5 \mathrm{~W})$. 
Conclusion: Despite the higher complexity and power dissipation, the (adaptive or reprogrammable) digital controller is the preferred solution. After all, it will be another 15 years before DARWIN will fly, and advances in digital processing are made at a rapid pace.

\section{DESIGN OF THE OPTICAL DELAY LINE}

The selected ODL concept consists of:

Optics: 2-mirror cat's eye

Guiding: $\quad$ Magnetic bearings constraining 5 DOF with eddy current sensors and central digital controller

Actuation: $\quad$ Single stage with voice coil actuator and digital controller.

\subsection{Optical Configuration}

The two-mirror cat's eye contains one parabolic focussing and one flat mirror. There is room for an additional metrology beam, should the DARWIN mission require this.

A cat's eye configuration is relatively insensitive to tip/tilt induced by the guiding mechanism. A tilt of $0.4 \mathrm{mrad}$ results in an output beam deviation of less than $0.2 \mu \mathrm{rad}$.

The cat's eye structure and mirrors will all be constructed from Al 6061. This makes the design fully athermal.

Aluminium mirrors are often plated with electroless Nickel and then polished. However, the difference in CTE between Aluminium and Nickel often causes differential thermal bending, which may result in large wavefront errors [13].

Differential bending can be minimised by the application of Alumiplate on the Aluminium mirrors. Both materials have virtually the same CTE. Alumiplate can be diamond turned to a very low surface roughness, and requires no additional polishing [14]. TNO TPD has extensive in-house experience with diamond turning and has already performed tests on 50 $\mathrm{mm}$ diameter flat Alumiplate samples. Achieved surface roughness is around $2-3 \mathrm{~nm}$ RMS.

A single layer of reflective Gold coating will applied in the advanced coating facility of the Centre Spatiale de Liege (CSL).

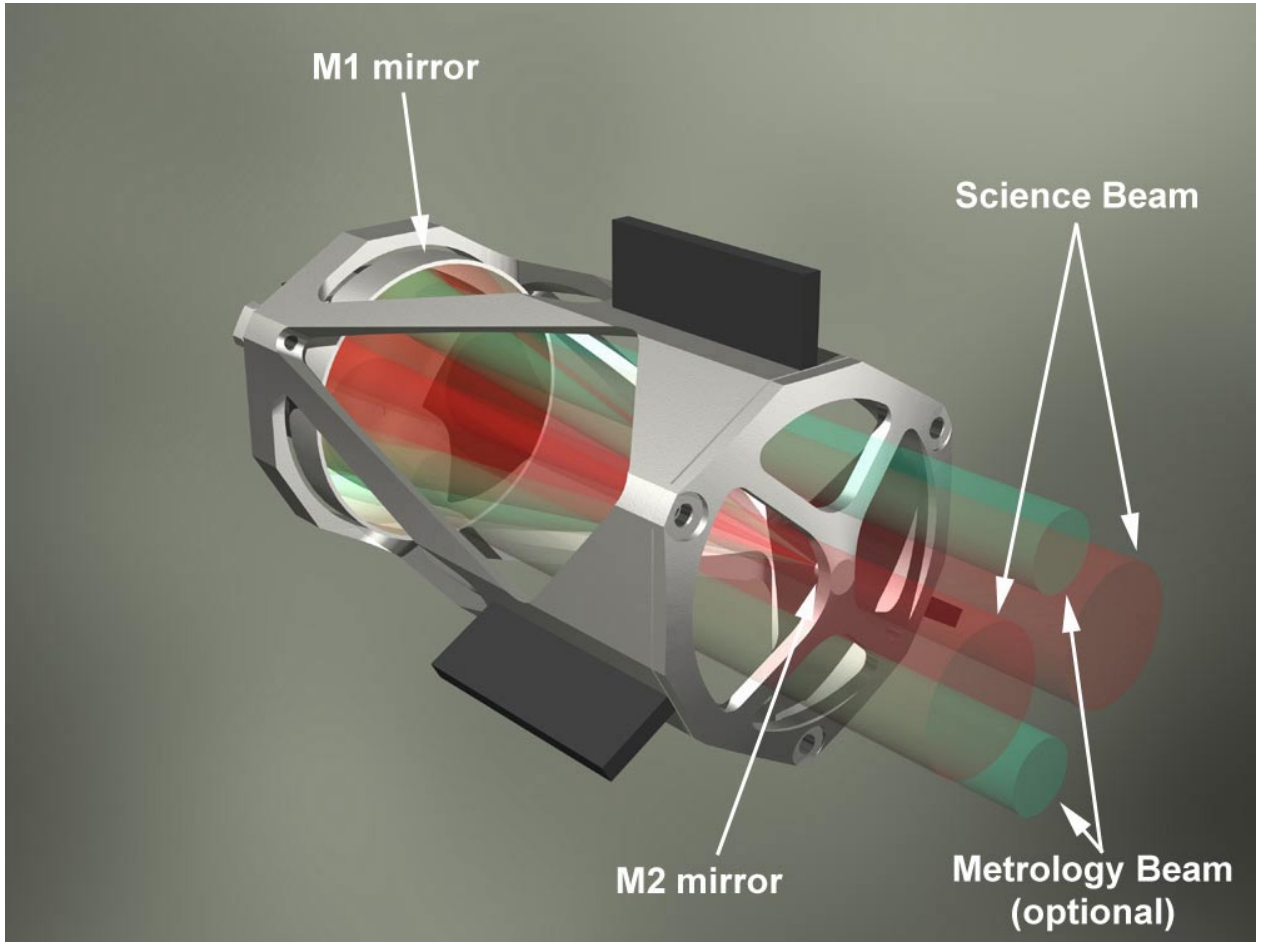

Fig. 5. ODL cat's eye 


\subsection{Guiding Mechanism}

The guiding mechanism is based on active magnetic bearings. The magnetic bearing system have been designed by Micromega, who have extensive experience with magnetic bearings, including space mechanisms such as MABE [17].

Magnetic bearings are relatively simple, and being contactless, do not require lubrication and do not wear. The inherent cleanliness makes them highly suitable for sensitive optical instruments.

In order to limit the power dissipation in the ODL, permanent magnet based reluctance force actuators will be used for the bearings.

Figure 6 shows the coplanar magnetic bearing configuration. A piece of soft iron is balanced between two permanent magnets. The system is inherently unstable. The permanent magnets are located in the middle of iron pieces. They produce a constant magnetic flux and the coils generate a variable magnetic flux that is added (or subtracted) to (from) the constant one. Eddy current sensors provide position information to the bearing controller, which in turn, keeps the soft iron centred.

Samarium Cobalt $\left(\mathrm{Sm}_{2} \mathrm{Co}_{17}\right)$ has been chosen as permanent magnetic material due to its relatively good stability at low temperature and its radiation resistance.

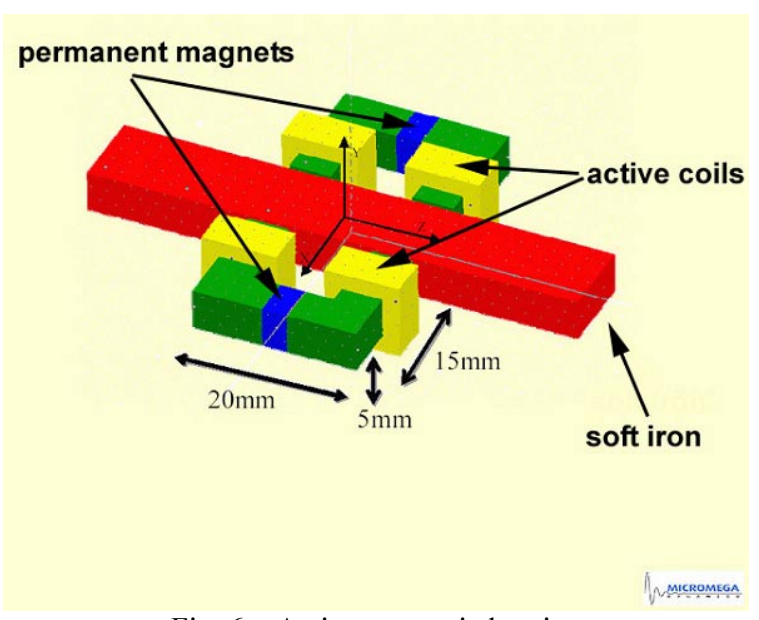

Fig. 6. Active magnetic bearing

The DARWIN BB ODL will have five magnetic bearings to constrain 5 degrees of freedom (the OPD controller constrains the other degree of freedom). A sixth bearing is added for redundancy. The system is capable of working with any one of the bearings switched off. In addition the coils are made redundant as well, for maximum system reliability.

Micromega has made a mathematical model of the ODL to optimise bearing layout and control bandwidth and calculate self induced perturbations.

The selection of the guiding bandwidth is driven by two contradictory requirements:

- Reducing the sensitivity of the mechanism to external perturbations, including actuator misalignments and ground acceleration, requires a high closed-loop bandwidth

- Reducing the self induced perturbation force, requires a low closed-loop bandwidth

In this trade-off the priority was put on reducing the sensitivity to external perturbations.

Subsequent analysis showed that the self induced perturbations of the magnetic bearings are below the expected FEEP induced perturbations of the DARWIN hub. 


\subsection{Actuation and OPD Control}

The design of the actuator (coil, magnet, electronics) is based on proven hardware used for the ISO mission and currently under construction for HIFI (both for a cryogenic environment). The actuator is being developed by SRON.

The voice coil actuator is located at the back of the cat's eye. The coil is attached to the static part of the ODL and the magnet is attached to the moving part, thus preventing disturbance forces caused by electrical wires. For reasons of redundancy, the voice coil consists of two concentric coils, an inner and an outer one. The selected magnet material is Samarium Cobalt $\left(\mathrm{Sm}_{2} \mathrm{Co}_{17}\right)$, because its behaviour at cryogenic temperatures can be predicted more accurately than e.g. NeFeBo.

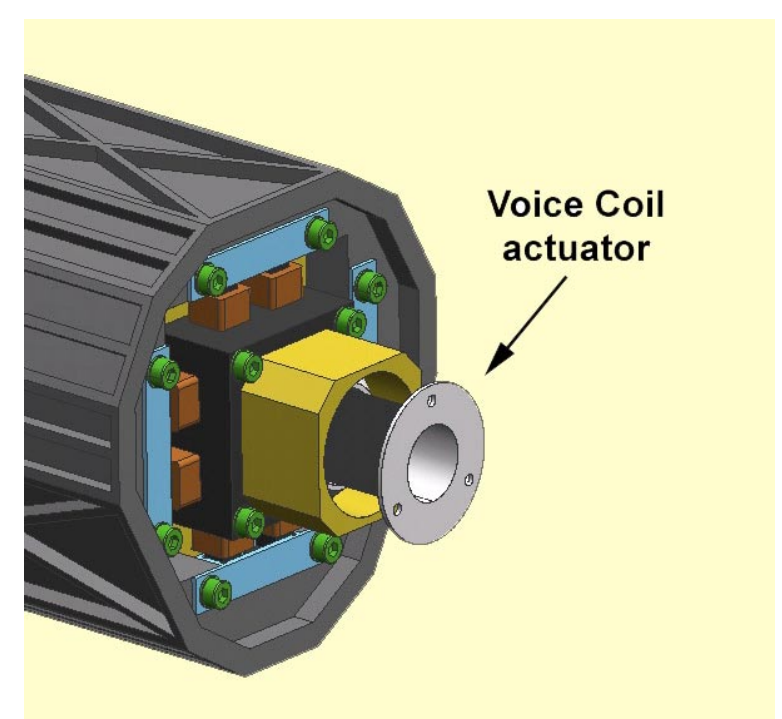

Fig. 7. ODL Voice Coil actuator

The DARWIN BB ODL will use a digital controller. The controller will be implemented on a real-time Linux PC to enable quick adjustment and fine tuning of control parameters during the development phase. For the DARWIN mission, the control algorithm will be implemented in a low power digital processor. The control model is shown in figure 8 .

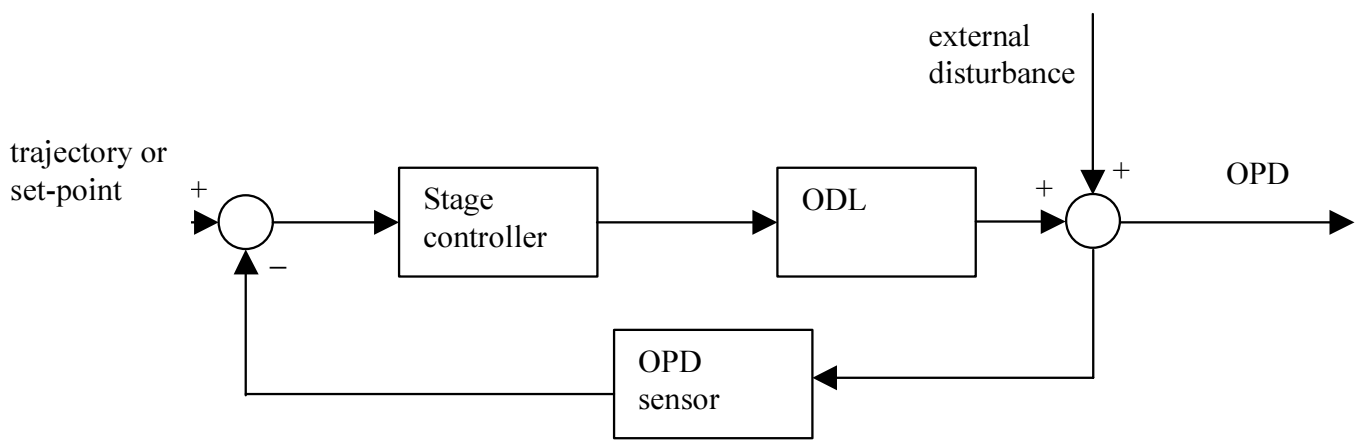

Fig. 8. OPD control model of single stage delay line 
The OPD sensor in DARWIN (Fringe Sensor) will be simulated by a laser metrology system with subnanometer resolution. This has the advantage that a large range of control bandwidths can be tested, e.g. to cope with a much higher disturbance spectrum during ground testing. With the advanced control algorithms that have been developed by TNO TPD during the passed decennium, a rejection ratio of upto 1:3.000 can be achieved, thus enabling nanometer stability, even in an environment with high frequency disturbance.

In path stabilization mode the control system needs to reject a stochastic-type disturbance by means of a feedback action (there is no advance information available). For this type of regulator system fundamental limits on the performance apply. The maximum, theoretical achievable disturbance rejection depends on the spectral characteristics of the disturbance together with the total loop delay. Here, we have assumed that the plant dynamics - other than the delay can be compensated for perfectly. In general, the maximum achievable disturbance rejection improves with a more narrowband disturbance spectrum and a smaller loop delay.

To illustrate the theoretical optimum performance, a simulation has been performed for rejection of the micro-vibration disturbance and a simplified plant model of the opto-mechanical structure only.

The influence of loop delay on controller performance is shown in figure 9.

Experimental results at TNO TPD and Dutch Space on other delay lines, show that the predicted rejection ratio is achievable.

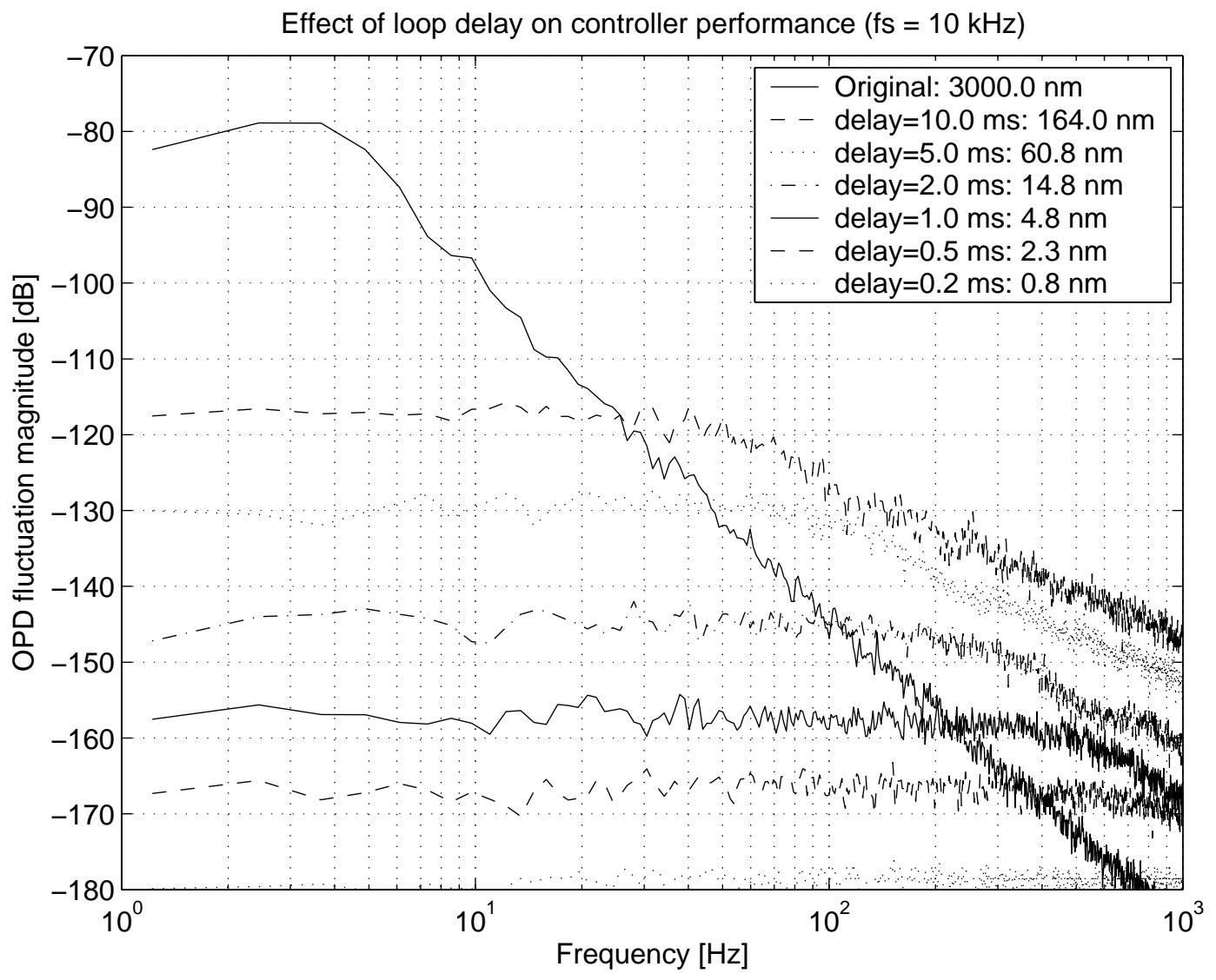

Fig. 9 Effect of loop delay on controller performance 


\section{CONCLUSION}

Analyses show that the design of the DARWIN BB ODL meets all ESA requirements. The use of a two-mirror cat's eye, magnetic bearing guiding and single OPD actuator, results in an extremely compact mechanism.

The DARWIN BB ODL is representative of a future flight mechanism, with all materials and processed used being flight representative.

The estimated mass of the ODL will be below $2 \mathrm{~kg}$ and the approximate envelope will be approximately $200 \times 100 \times 100$ $\mathrm{mm}$ (100 $\mathrm{mm}$ less than the ESA requirement). Overall electrical power dissipation will be well below $2.5 \mathrm{~W}$, and the electrical power dissipation on the optical bench will be considerably below the maximum allowable $25 \mathrm{~mW}$.

The ODL assembly is shown in figure 10.

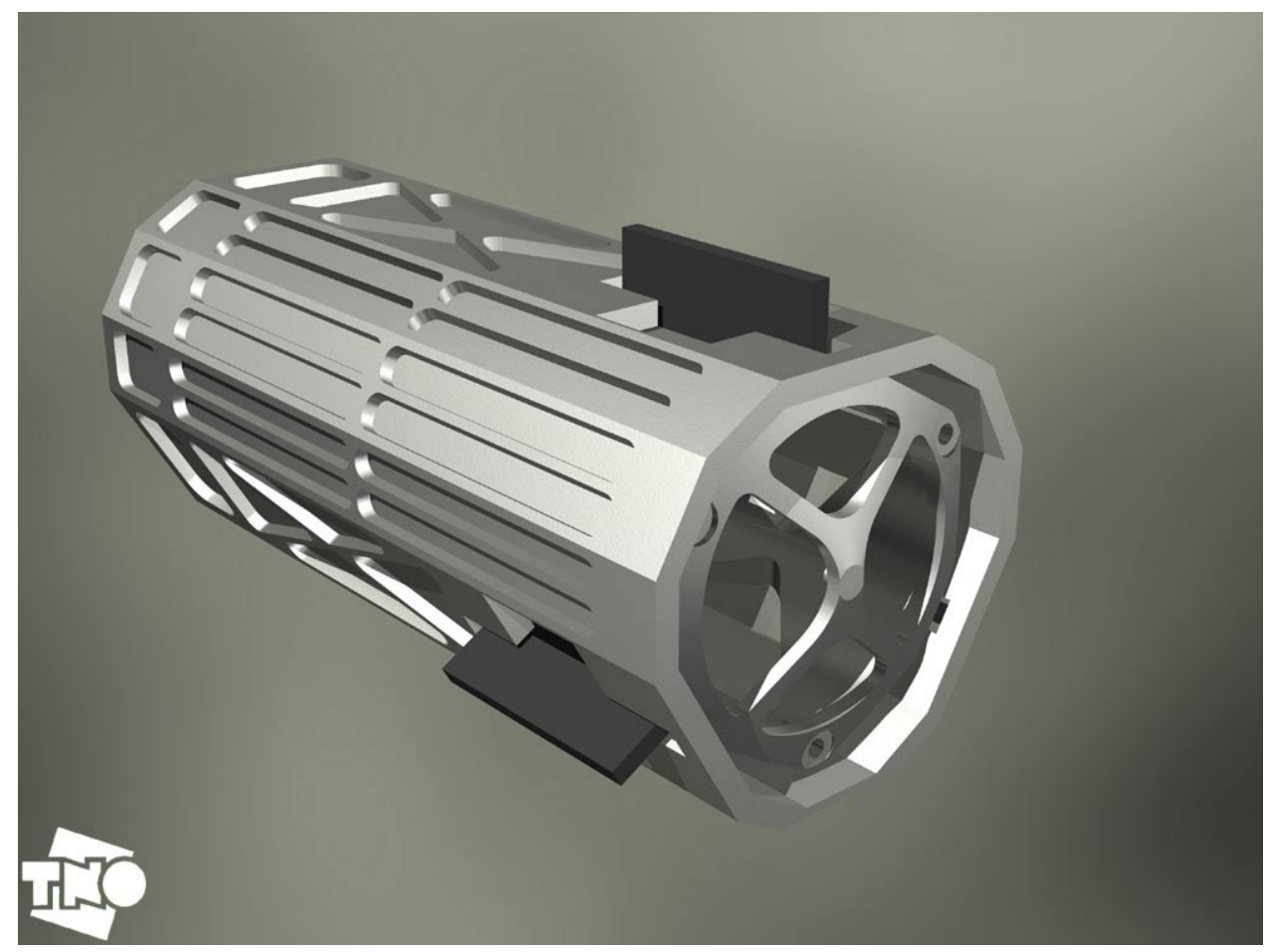

Fig. 10. DARWIN BB ODL assembly

\section{AKNOWLEDGEMENTS}

TNO TPD would like to thank Josep Maria Perdigues Armengol (ESA-ESTEC), Joost Carpay (NIVR) and Gustavo Vallejo (Alumiplate) for their support during the development of the delay line. 


\section{REFERENCES}

[1] Statement of Work, Optical Delay Lines, Programme Reference: TRP, ID-OP-12 TOS-MMO/2002/276, issue 2.0, 6 March 2003.+ clarifications 1, 2 and 3

[2] DARWIN-The InfraRed Space Interferometer, Concept and Feasibility Study Report", Version 1.2 ESASCI(2002)12, April 2002

[3] B. Snijders et al., "Free-beam delay line for a multi-aperture optical space interferometer stabilized on a guide star", SPIE Vol. 2209, 1994

[4] B.C.Braam et al., "Kinematic six-ball guide for long stroke optical delay line", Proc. Sixth European Space Mechanisms \& Tribology Symposium, Zurich (Switzerland), 1995, ESA SP-374, August 1995

[5] R.J. Calvet et al., 'Enabling design concepts for a flight-qualifiable optical delay line', SPIE Vol. 3350 (1998), pp. 35-47

[6] S. Mekid and M. Bonis, Conceptual design and study of high precision translational stages: application to an optical delay line, Precision Engineering (1997), Vol. 21, No. 1, p. 29 - 42.

[7] 'Optical Delay Line Nanometer Level Pathlength Control Law Design For Space-Based Interferometry', Robert L. Grogan, Gary H. Blackwood, and Robert J. Calvet. SPIE, Kona, Hawaii, March 1998, pp.14-25

[8] M.R. Swain, P.R. Lawson, J.D. Moore, and D. Jennings, 'Cryogenic delay line for far-IR interferometry in space', 36th International Astrophysical Colloquium, 2-5 July 2001, Liege, Belgium.

[9] Y. Honma et al., 'Development of the fine delay line in the Mitaka optical and infrared array (MIRA) project', SPIE Vol. 3350 (1998), pp. 192 - 201

[10] 'Active Vibration Control for an Optical Delay Line', N.J. Doelman, T.C. van den Dool. Proc. of Active 2002, pp.887-898

[11] B. Koehler et al., 'Optical delay line for stellar interferometer', SPIE Vol. 1236 (1990), pp. 463 - 472.

[12] F. Derie et al., 'VLTI delay lines: design, development, performance results, and commissioning on site', SPIE Vol. $4006(2000)$.

[13] Improved cryogenic aluminum mirrors, D. Vukobratovich, K. Don, R. E. Sumner, National Optical Astronomy Observatories, SPIE Vol. 3435-02 (1999)

[14] Diamonds turn infrared mirrors smooth, D. Vukobratovich, K. Don, R. E. Sumner, National Optical Astronomy Observatories, Optoelectronics World, October 1998

[15] "DSP (Demanding Space-Based Processing!) The path behind and the road ahead" - Dr SM Parkes Applied Computing, University of Dundee, Dundee, DD1 4HN, Scotland, UK

[16] A. Molenaar, H.F. van Beek, and M.J.L. Sanders, " A New Linear Magnetic Bearing Configuration for High Accuracy Positioning", in Proceedings of MAG97: Magnetic Bearings, Magnetic Drives and Dry Gas Seals Conference \& Exhibition (P. Allaire, ed.), (Alexandria, Virginia), pp. 313-322, Technomic Publishing Company, Inc, July 1997

[17] MABE, Fine Precision Mechanism Based on Magnetic Bearing Technology, Micromega Dynamics - Executive Summary (ESA Contract No 13676/99/NL/PA) 